\title{
A Monolithic Three-Dimensional Ultrasonic Transducer Array for Medical Imaging
}

\author{
Jingkuang Chen, Xiaoyang Cheng, I-Ming Shen, Jian-Hung Liu, \\ Pai-Chi Li, Senior Member, IEEE, and Mengli Wang
}

\begin{abstract}
This paper presents the first monolithic multidirection-looking ultrasonic imager for minimally invasive medical diagnosis. In contrast to the traditional planar ultrasonic imagers that can only view in one direction, this 3-D array is able to view in multiple directions by using seven planar imagers integrated on a hexagonal silicon prism. Each facet on the prism is integrated with a planar 1- or 2-D capacitive-micromachined ultrasonic-transducer imager array for viewing in a specific direction. Each facet is connected by a flexible dielectric membrane, which is monolithically fabricated with the transducers. The dielectric membranes also support the thin-film electrical interconnects between the arrays on different facets. The substrate is folded into a hexagonal prism after completion of the transducer microfabrication process. With this architecture, a one flip-chip bonded or monolithically integrated front-end electronic circuit will be able to manage all the imagers on the 3-D array. The number of bonding wires for a connection to external electronics can therefore be reduced. Imager prisms, which are ranging from 1 to $4 \mathrm{~mm}$ in diameter and 2 to $4 \mathrm{~mm}$ in length, and positioned to view in seven directions, have been prototyped. Preliminary testing shows that the imager transducers behaved consistently before and after the assembly process. Applications of this 3-D imager array include capsule ultrasound endoscope, intravascular ultrasound, and other internal imaging needs.

[2006-0269]
\end{abstract}

Index Terms-Acoustic transducers.

\section{INTRODUCTION}

$\mathbf{E}$ VOLVED from sonar technology, ultrasound imaging [1]-[3] has been widely used for medical diagnosis since the early 1970s. In this imaging process, high-frequency acoustic waves interact with biological tissue, so that the images of anatomical structures inside the body can be revealed in real time from the reflected ultrasonic pulses. Compared with other medical imaging techniques (X-ray, MRI, CT, etc.) [4], [5], the advantages of ultrasonic imaging include nonradioactivity, real-time acquisition, affordable equipment cost, and feasibility for the miniaturization in minimally invasive applications. In

Manuscript received December 3, 2006; revised May 10, 2007. Subject Editor A. Ricco.

J. Chen, X. Cheng, and M. Wang are with the Department of Electrical and Computer Engineering, University of New Mexico, Albuquerque, NM 87131 USA (e-mail: jingkuang@yahoo.com; jingchen@ece.unm.edu; xycheng@unm.edu; mengwang@unm.edu).

I.-M. Shen and J.-H. Liu are with the Department of Electrical Engineering, National Taiwan University, Taipei 10617, Taiwan, R.O.C. (e-mail: r94921053@ntu.edu.tw; d92921029@ntu.edu.tw).

P.-C. Li is with the Department of Electrical Engineering and the Graduate Institute of Biomedical Electronics and Bioinformatics, National Taiwan University, Taipei 10617, Taiwan, R.O.C. (e-mail: paichi@cc.ee.ntu.edu.tw).

Color versions of one or more of the figures in this paper are available online at http://ieeexplore.ieee.org.

Digital Object Identifier 10.1109/JMEMS.2007.902449 addition to laboratory-scale platforms scanning from outside the body, the ultrasonic transducer array has been packaged into probes for use in invasive applications such as tubular ultrasonic endoscopes [6] for the detection of tumors in the gastrointestinal tract or the intravascular-ultrasonic (IVUS) [7] imager for the examination of plaque [8] in a blood vessel. Traditionally, piezoelectric ceramics, e.g., lead zirconate titanate (PZT) [9], [10], have been the most widely used materials in fabricating medical ultrasonic transducers. The piezoelectric ceramics contract and expand upon application of electrical voltages. This voltage-induced deformation at ultrasonic frequencies will agitate the ambient media and emit ultrasound. Working as an ultrasonic receiver, the piezoelectric ceramics deform upon reception of ultrasonic waves. The deformation induces an electrical voltage across the ceramics due to piezoelectricity. This electrical signal reveals the strength, frequency, and phase of the ultrasounds. These piezoelectric ultrasonic transducers are generally arranged as 1-D [11] arrays. With the increasing demand for real-time 3-D images in medical diagnosis, many efforts have been attempted to build 2-D [12], [13] piezoelectric ultrasonic arrays. Although initial success in such efforts has been achieved, and some commercial devices have been introduced, several technical obstacles such as electrical impedance match [14] and interconnection [14] still present challenges to engineers working in this field. Due to the small size and the material properties of the 2-D piezoelectric ultrasonic-transducer elements, their electrical impedances are much higher than that of the control circuitry. This electrical impedance mismatch degrades the receiving sensitivity and transmitting efficiency of the transducers.

Another problem associated with the piezoelectric ultrasonic transducers is that the acoustic impedance [15] of piezoelectric thin films does not match with that of the fluids encountered in many applications. The problem of impedance mismatch grows worse with gaseous fluids. The acoustic impedance of PZT, water, and air is 30 Mrayl $\left(10^{6} \mathrm{~kg} / \mathrm{m}^{2} \mathrm{~s}\right), 1.5 \mathrm{Mrayl}$, and 400 rayl $\left(\mathrm{kg} / \mathrm{m}^{2} \mathrm{~s}\right)$, respectively. With such a significant impedance mismatch, a large percentage of the ultrasound energy would bounce back at the transducer-fluid interface, and the acoustic bandwidth of the transducer would be relatively narrow. Although special designs have added impedance matching layers [15] on the transducer surface to alleviate the problem, the poor coupling efficiency from the transducer to tissue is still a fundamental limit of piezoelectric ultrasonic systems used in medical diagnosis. Due to problems with acoustic impedance mismatching, high operation voltage, ultrasound-signal bandwidth, surface displacement, and electrical interconnect/impedance 
match in controlling the electronics of piezoelectric ultrasonic transducers that constrain the system design, other technologies are being explored. Since 1989, there have been many efforts in the development of capacitive micromachined ultrasonic transducers (CMUTs) [16]-[18]. A capacitive ultrasonic transducer is composed of a suspended membrane and a counter electrode fixed to a supporting substrate. When an ac signal is applied between the membrane and the fixed electrode, the alternating Coulomb force drives the membrane to vibrate and generate the ultrasonic waves. At the same time, the reception of impinging ultrasound waves will cause the membrane to deform and change the capacitance. Constructed using the technology developed in manufacturing integrated circuits, these micromachined ultrasonic elements have been fabricated in 1- and 2-D arrays and are compatible with the integration of front-end electronics. As opposed to the piezoelectric ultrasonic devices, which require a thick matching layer of a millimeter for lowfrequency operations, the micromachined ultrasonic devices are made of thin films, which, typically, have a thickness ranging from submicrometers to a few micrometers. By adjusting the geometrical dimension of the membranes, a broad spectrum of ultrasounds can be transmitted and sensed by devices fabricated on the same substrate using the same microfabrication process.

For imagers operating outside of the body, the ultrasound imaging near the surface of the body is capable of resolutions on the order of several hundred micrometers [19]. As the penetration depth of ultrasounds decreases with shorter wavelengths, the imaging of anatomic structures inside the body requires the use of longer wavelength ultrasounds. These longwavelength ultrasounds degrade the image resolution. Since short-wavelength ultrasounds cannot penetrate through a thick biological structure, a straightforward approach in acquiring high-resolution images of anatomical structures deep inside a biological object is to place an ultrasound imager inside an organ or tissue to acquire the images from a nearby position. In order to minimize the issue disruption caused by the introduction of the ultrasonic imager, these tools need to be as small as possible. The operation voltage and power of these invasive devices should be low to minimize electrical and thermal disturbance to the biological object being diagnosed. Additionally, the surface of such devices should be passivated with biocompatible materials to prevent any unexpected chemical reactions in the tissue.

Conventional piezoelectric ultrasonic transducers and CMUT are built on a bulky ( $>500-\mu \mathrm{m}$ thick) piece of silicon or other substrate and arranged in 1- or 2-D array [16]-[18] for medical imaging. Most of these ultrasonic imagers are only able to view in one direction. One of the exceptions is the piezoelectric side-viewing imager used in IVUS [7]. IVUS was the first commercially available clinical tool with the capability of insertion into a blood vessel for internal imaging. Intravascular ultrasound uses a long flexible tube called a catheter, which is typically $0.5-3 \mathrm{~mm}$ in diameter, to support an imager probe at its tip, from which the ultrasound signals are transmitted and received. Currently, there are two kinds of IVUS commercially available. The first kind is made of a 1-D array of piezoelectric transducers mounted around a catheter. These transducers can be electronically scanned and acquire 2-D images around the

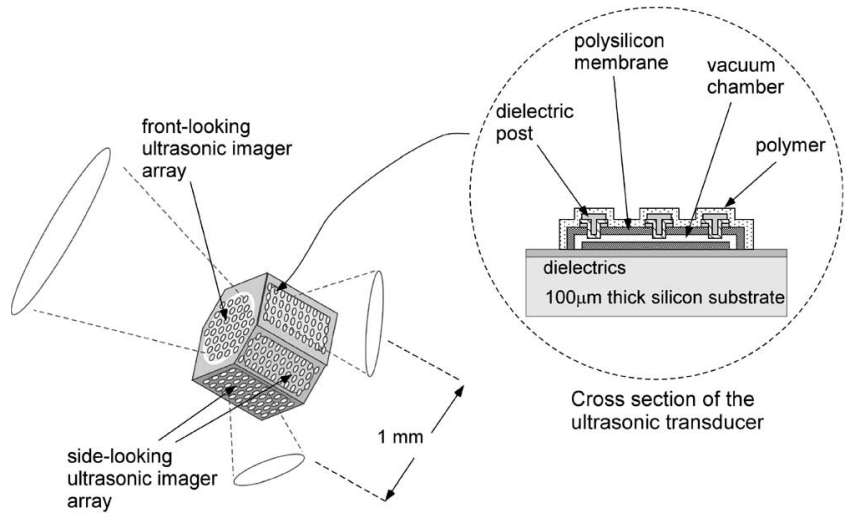

Fig. 1. Perspective of the monolithic 3-D ultrasonic transducer array for multidirection ultrasonic imaging.

catheter. The second kind is equipped with a single ultrasonic transducer, which mechanically rotates around the catheter. The single-transducer IVUS provides similar imaging capabilities as the 1-D array. With its unique capability of shooting close-up images from the inside of a blood vessel, intravascular ultrasound is able to identify problems that other tests cannot adequately reveal and has become a popular tool in diagnosing vessel blockage and other abnormalities. Similar ultrasonic tools have been used for other endoscopic applications, including diagnosing diseases of the urinary organs and the gastrointestinal tract [20]. However, since transducers are either mounted on the sidewall or rotating around the catheter, these commercial IVUS heads are capable only of imaging on planes perpendicular to the direction of the blood flow.

Due to the incapability of Doppler-based blood flow measurement and guiding intervention of the commercial sideviewing IVUS, recent efforts have been made to develop a front-looking IVUS. Oralkan et al. [21] and Knight and Degertekin [17] have demonstrated the feasibility of implementing front-looking IVUS through the use of annular CMUT arrays mounted on the front end of a catheter. These microelectromechanical-system (MEMS)-based IVUSs are restricted to only the front view and are not equipped with sidelooking imagers. In a parallel effort, O'Donnell [22], [23] proposed a design that divides a 1-D array of piezoelectric transducers into two sections. The shorter section is bent and mounted on the front end of the catheter to form an annular array for front looking, while the longer section is mounted on the sidewall of the catheter for side looking. This device, therefore, is potentially able to provide views from both the front and sides. Since the front- and side-looking transducers are of a different thickness after a special arrangement of electrical connections, the front- and side-looking imagers can operate at different frequencies. However, due to numerous elements missing in the front-looking imager, the prototype was not yet able to provide clinical imaging [23].

This paper reports the design, fabrication, and characterization of a MEMS-based 3-D ultrasonic transducer array for medical imaging. On this 3-D platform, multiple ultrasonic transducer arrays are monolithically integrated on a flexible silicon substrate, which is then assembled into a 3-D prism. Each facet on this silicon prism is equipped with a single ultrasonic 


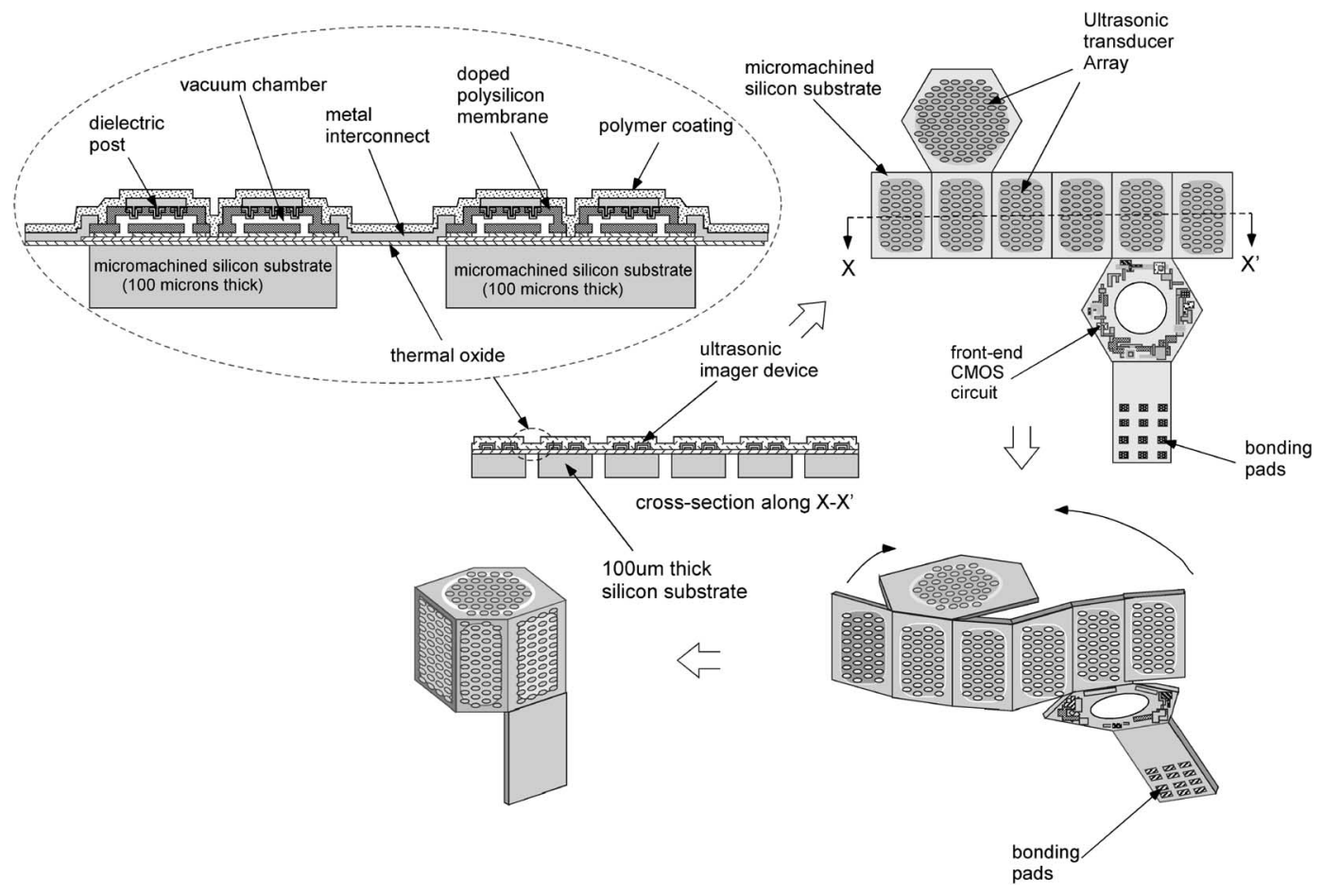

Fig. 2. Assembly of a planar monolithic transducer array into a 3-D imager prism for viewing at multiple directions.

imager array for viewing in a specific direction. Connected by thin-film structures monolithically fabricated with the transducer, this 3-D imager array can be managed by one front-end electronic chip, which is either monolithically integrated or flipchip bonded, reducing the need for bonding wire in connecting to the external electronics. This monolithic architecture is also easier to assemble into a 3-D array than multiple pieces of separate imager chips. A seven-direction-looking IVUS head has been prototyped with this approach, in which one frontlooking annular array and six-side-looking arrays are integrated on one miniature platform suitable for internal imaging.

\section{THREE-DIMENSIONAL ULTRASONIC-TRANSDUCER DESIGN}

Fig. 1 shows the schematic of this monolithic 3-D transducer array for ultrasonic imaging. As shown in the inset of Fig. 1, suspended thin-film membranes made of doped polysilicon are used in transmitting and receiving ultrasounds. Multiple planar arrays of these drum-shaped transducers are integrated on the surface of a miniaturized silicon prism for viewing in different directions. The six-side-looking array can cover a full $360^{\circ}$ viewing angle around the side direction. Unlike the traditional ultrasonic transducers, which are normally integrated on a thick $(>500 \mu \mathrm{m})$ substrate, this multidirection-looking imager is built on a silicon substrate, which is micromachined to be approximately $100-\mu \mathrm{m}$ thick. A thinner substrate helps in reducing the overall size of this 3-D imager head while also decreasing the substrate acoustic crosstalk [24] between the transducers. Acoustic crosstalk refers to the phenomenon of interference caused by the transference of energy from a neighboring transducer such as leakage or coupling, which results in unwanted acoustic signals.
As shown in Fig. 2, the silicon substrate is divided into multiple small plates, each containing an imager array. The number of ultrasonic-transducer elements on the front-looking annular arrays typically ranges from 42 to 64 . The 1-D sidelooking arrays typically contain 15-25 elements, while the 2-D side-looking arrays typically contain 100-196 elements. A flexible dielectric membrane monolithically fabricated with the transducer connects the imager plates to each other. The dielectric membranes also support the thin-film electrical interconnects between the imager plates. After the completion of the transducer fabrication process, the substrate is folded and assembled into a 3-D prism, onto which each facet is equipped with one planar imager array for viewing in a specific direction. Although a seven-imager device is illustrated, this technology allows implementation of a 3-D array with any reasonable number of imagers. After the array is assembled into a 3-D prism, epoxy or other biocompatible polymers are used to fill into the cavity inside the prism, so the whole structure is glued together. The polymer also provided the mechanical support for this imager so that it will not easily crash from external pressure.

\section{FABRicAtion Process}

The fabrication of this 3-D imager array started with a ptype 4-in (100) silicon wafer. The surface of the wafer was first doped with phosphorus, using a diffusion process at $950{ }^{\circ} \mathrm{C}$ for $60 \mathrm{~min}$ to form a highly conductive surface area. This area was used in reducing charge feed-through for the operation of electrostatic devices to be built on the wafer. One-micrometer thick of silicon dioxide was next grown thermally, followed by a deposition of $5000 \AA$ of silicon nitride and $250 \AA$ of 


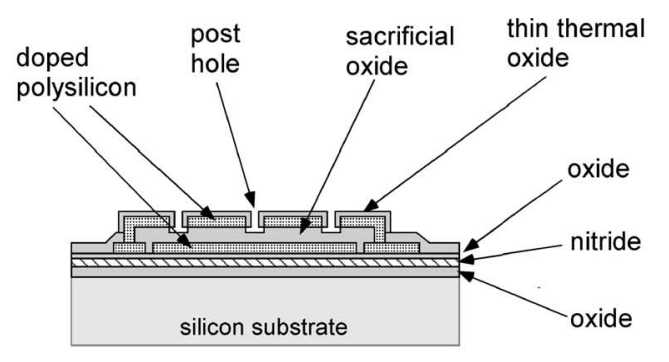

(a)

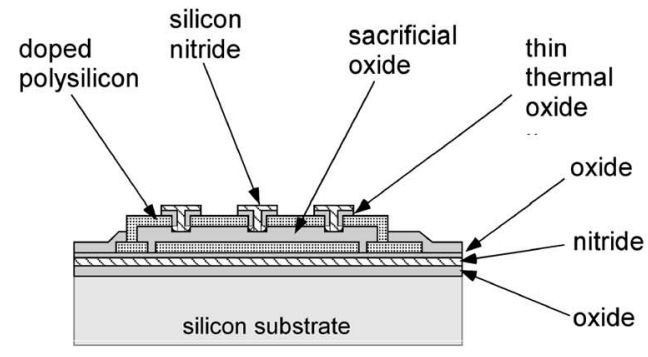

(b)

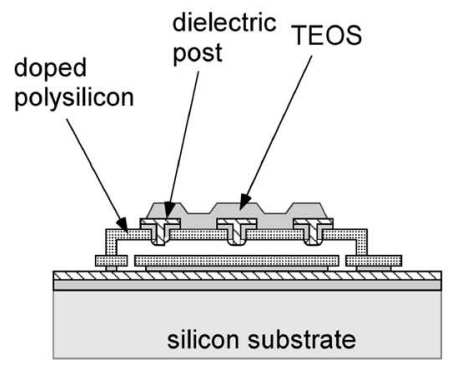

(c)

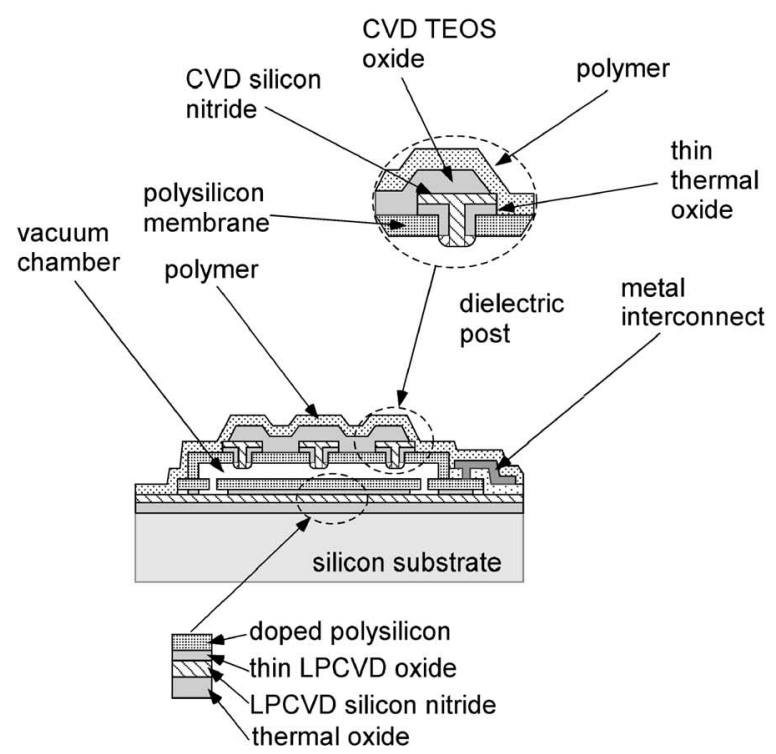

(d)

Fig. 3. Fabrication sequence of the MEMS ultrasonic transducer. (a) After a thin layer of thermal oxide is grown on top of the patterned polysilicon membrane. (b) After the deposition and patterning of the LPCVD silicon nitride. (c) After the deposition and patterning of TEOS. (d) After the passivation with a polymer layer.

silicon dioxide using low pressure chemical vapor deposition (LPCVD). The purpose of the top thin silicon dioxide was used to separate the silicon nitride film from the polysilicon microstructure. Using LPCVD, $5000 \AA$ of polysilicon was then

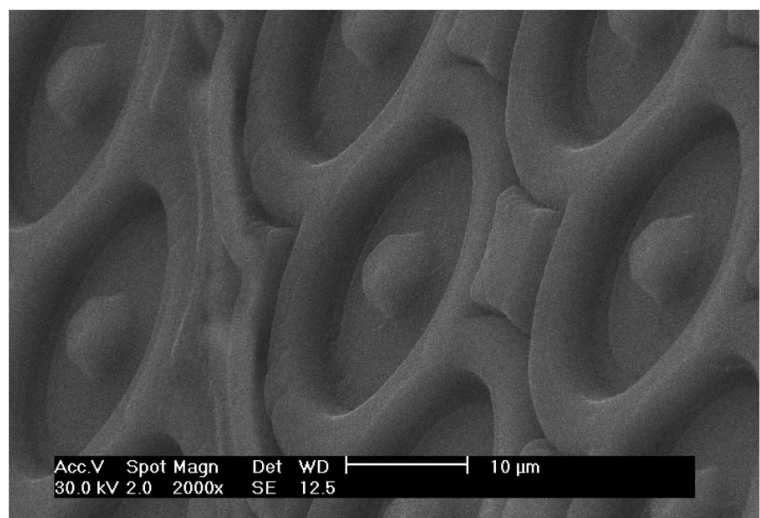

Fig. 4. SEM photograph of the transducer after the sealing TEOS was patterned.

deposited and doped with phosphorus using a diffusion process at $950{ }^{\circ} \mathrm{C}$ for $30 \mathrm{~min}$. The sheet resistance of the polysilicon film was $10-12 \Omega /$ square after diffusion and postannealing. The polysilicon film was patterned using a photolithography process and a reactive ion etching to form electrodes, electrical interconnects, and anchoring pads for the polysilicon structures to be built in the subsequent step. A thin layer of phosphosilicate glass was next deposited using the LPCVD. The typical thickness of this sacrificial oxide was $2000 \AA$ after annealing. The sacrificial oxide was patterned using a photolithography process and reactive ion etching to form anchoring holes. A layer of $1.5 \mu \mathrm{m}$ of polysilicon film was next deposited using the LPCVD and doped with phosphorus, using a diffusion process at $950{ }^{\circ} \mathrm{C}$. The polysilicon film was annealed in nitrogen gas at $1100{ }^{\circ} \mathrm{C}$ for $60 \mathrm{~min}$ for stress relief. The stress of the structural polysilicon film was between -5 and $-20 \mathrm{MPa}$ after the annealing process. A photolithography process and a reactive ion etching were used to pattern the structural polysilicon. This step defined the shape of the polysilicon membranes while simultaneously carving an array of $2 \times 2 \mu \mathrm{m}$ holes in the polysilicon membrane. The holes in the membrane will harbor an array of dielectric posts in the polysilicon membrane as a mechanical stop. Without these dielectric posts, the polysilicon membrane could short to the counter electrode during transducer operation. The dry etching that is used to pattern the structural polysilicon intentionally overetched into the sacrificial oxide for $200-300-\AA$ deep. The dielectric post molded by these pits will therefore protrude the same distance down from the lower surface of the polysilicon membrane. A thermal oxidation was then used to convert the surface of the polysilicon membrane into a thin $(\sim 250 \AA)$ layer of silicon dioxide, as shown in Fig. 3(a). Next, $2000 \AA$ of silicon nitride was deposited using the LPCVD. This layer and the thin thermal oxide underneath were patterned using the same mask, as shown in Fig. 3(b). The wafer was next etched in $49 \% \mathrm{HF}$ in removing the sacrificial oxide, freeing the membrane structures. A supercritical drying process was used after the release etching to prevent the stiction problem. The sacrificial release process slightly undercuts the thin silicon oxide layer under the polysilicon microstru ctures. It also undercuts the thin thermal oxide sandwiched between the top nitride layer and the polysilicon membrane around the dielectric posts. Due to the very thin $(\sim 250 \AA)$ oxide used in 


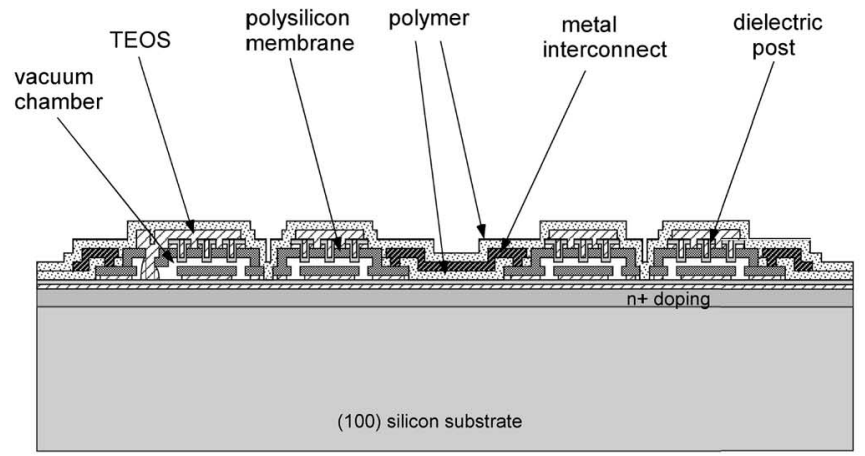

(a)

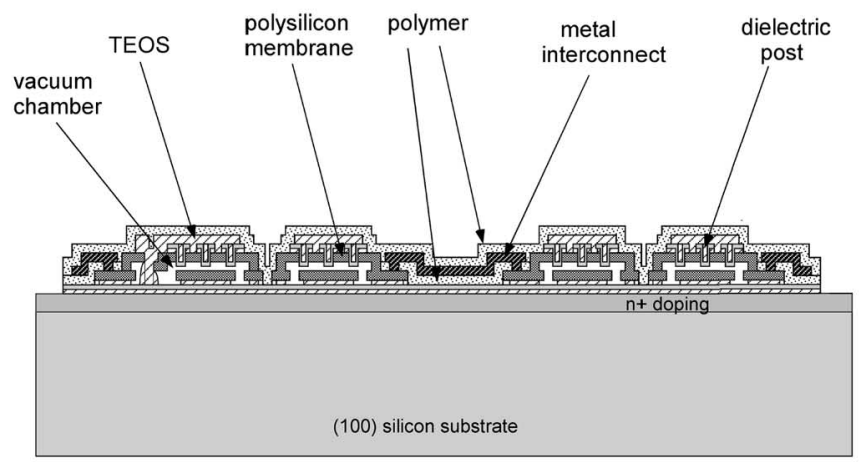

(b)

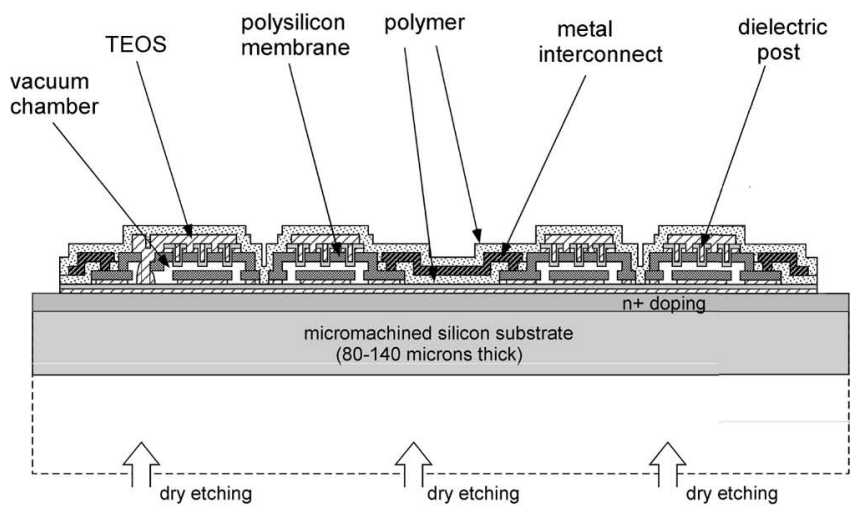

(c)

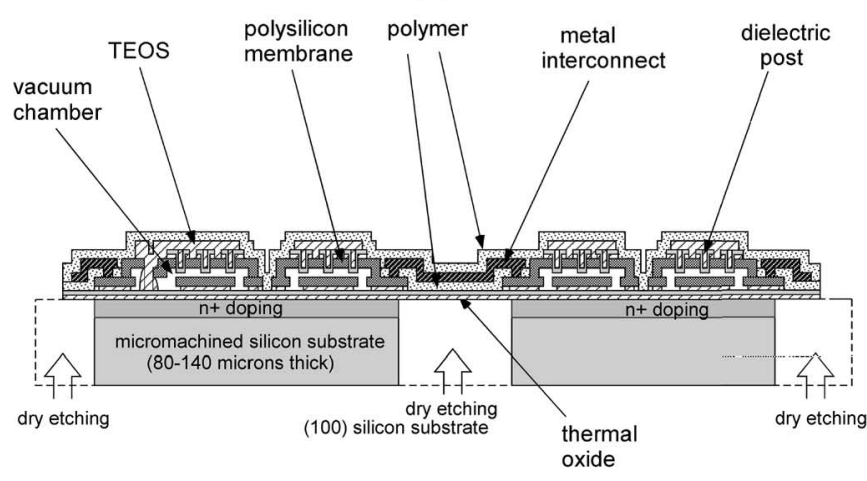

(d)

Fig. 5. Sequence in micromachining the silicon substrate. (a) After the completion of surface-micromachined transducer structures. (b) Five-micrometerwide trench is opened around the peripheral of the device. The dielectrics in this trench are completely removed using a dry etching. (c) Wet or dry flood etching from the backside is used to thin the wafer down to approximately $100 \mu \mathrm{m}$. (d) Wafer is patterned from the backside using a photolithography process and a deep silicon etching. This process forms flexible dielectric membranes between the device plates and releases individual devices from the substrate.

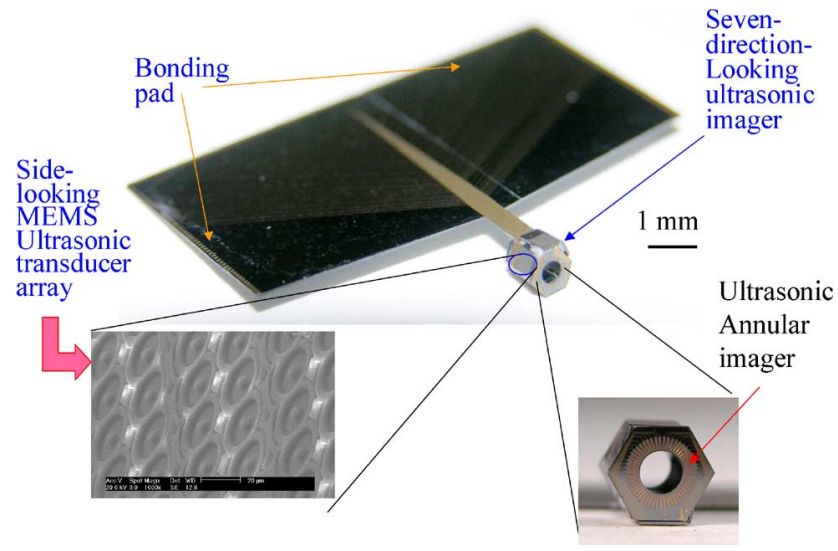

Fig. 6. Monolithic IVUS imager with the front- and side-looking imagers integrated on a hexagonal silicon prism. The diameter of the front-looking annular imager is $1 \mathrm{~mm}$. The circular hole in the middle of the front-looking imager is for the guiding wire.

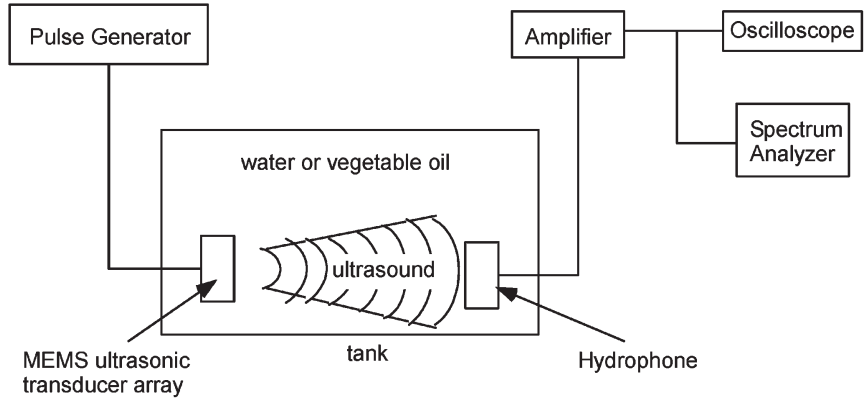

Fig. 7. Setup for transmission experiment.

this sandwiched structure, which limited the diffusion of the etchant into/out from a very narrow gap, these undercuts were insignificant and did not degrade the anchor structures. The wafer was then coated with a $3 \mu \mathrm{m}$ of PECVD tetraethoxysilane (TEOS). This TEOS layer was used to seal the release holes, such that the chamber under the membrane is kept at vacuum. A photolithography process and a wet etching were used to pattern this TEOS layer, leaving only the TEOS around the release hole and on the center of the membrane, as shown in Fig. 3(c). Fig. 4 is a SEM photograph of the transducer array after the sealing TEOS was patterned.

A 1- $\mu \mathrm{m}$-thick layer of parylene $\mathrm{C}$ was coated next by thermal evaporation. This layer was used in separating the metal interconnects from the silicon nitride. The parylene $\mathrm{C}$ film was patterned using a photolithography process and a reactive ion etching, which opens via holes on the metal-polysilicon connection area. After another photolithography process defined the metal layer pattern, $4000 \AA$ of aluminum was sputtered and patterned using a lift-off process. A total of $3 \mu \mathrm{m}$ of parylene $\mathrm{C}$ is next coated using thermal evaporation and then patterned. This patterning process exposes metal on the intended bonding pad area. Fig. 3(d) shows the cross section of the ultrasonic transducer after the parylene $\mathrm{C}$ coating.

After completion of the surface micromachining processes, as shown in Fig. 5(a), a photolithography process was used to define a 5- $\mu \mathrm{m}$-wide window around the peripheral of each device. The dielectric layers in this window are completely 

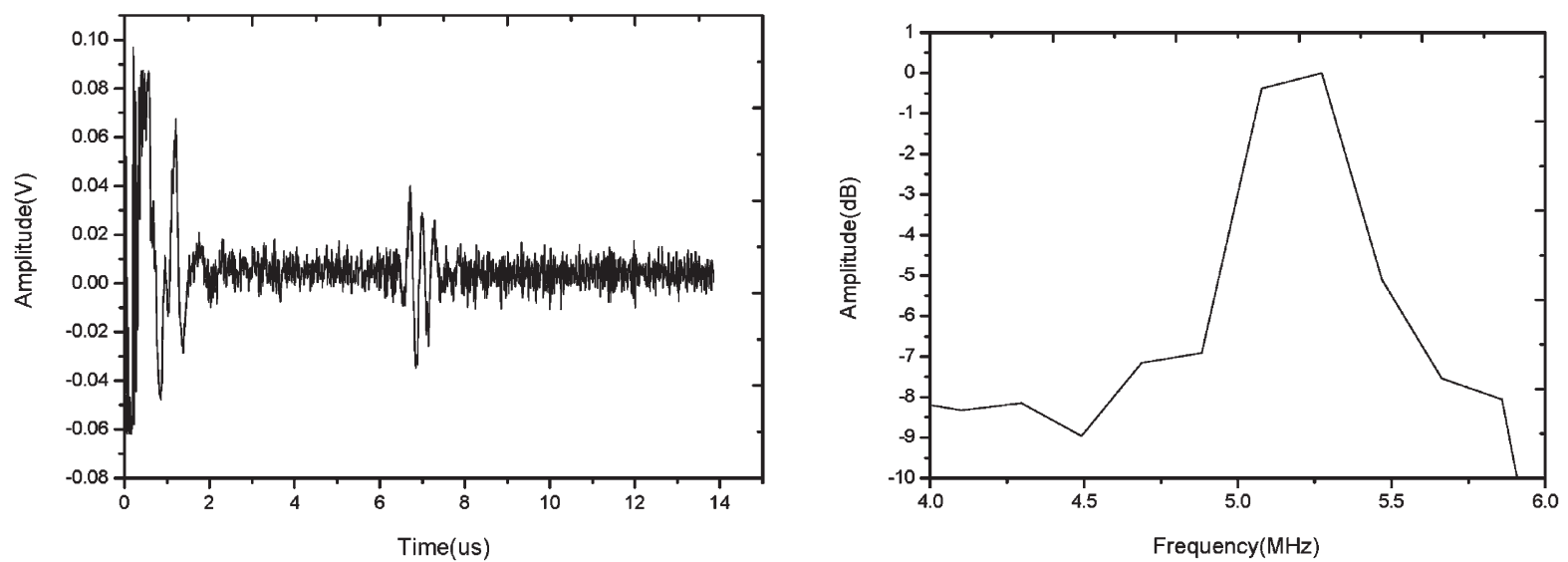

(a)
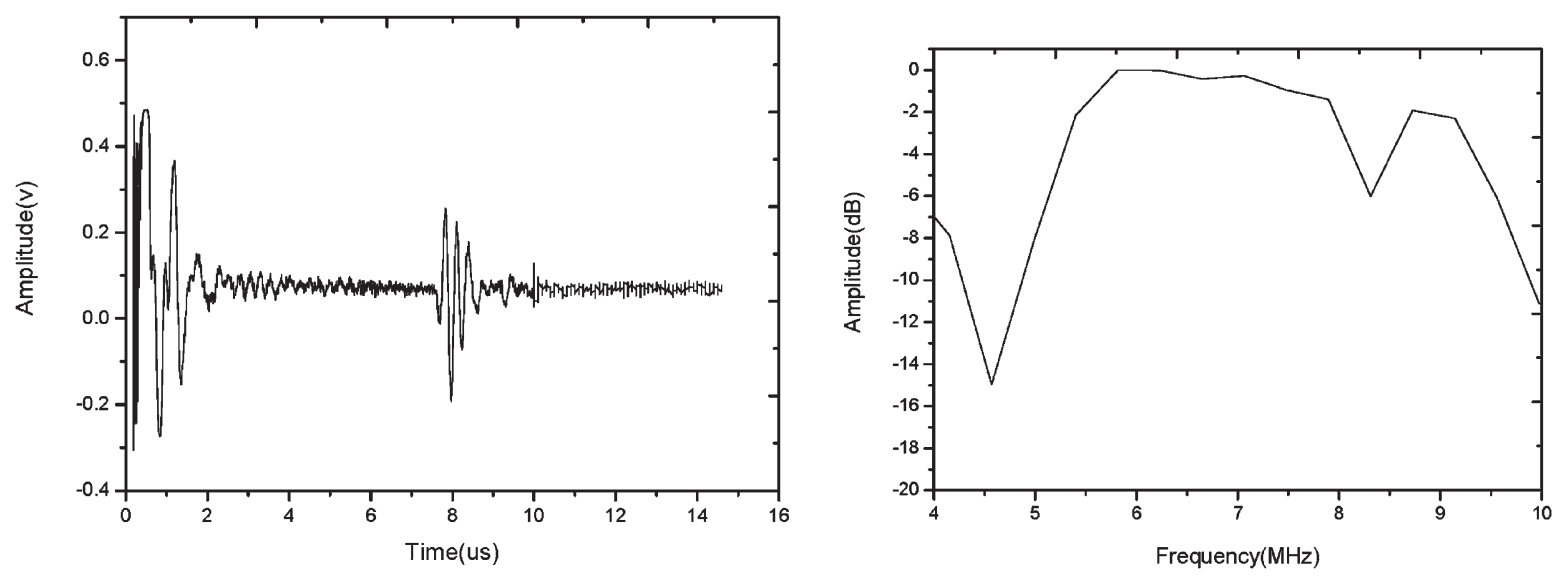

(b)
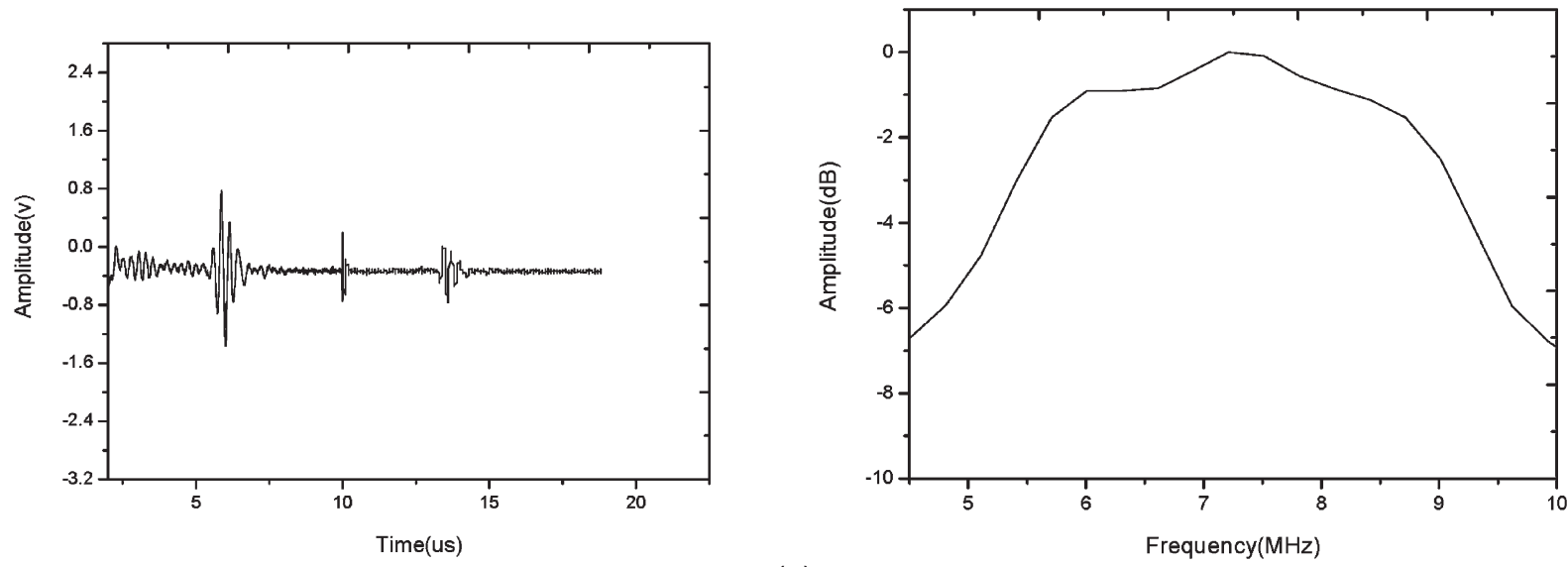

(c)

Fig. 8. Ultrasound signal transmitted by a 46- $\mu$ m-diameter transducer: (a) in water, (b) in water with the transducer which is coated with a thin layer of vegetable oil, and (c) in vegetable oil.

removed using a reactive ion etching, as shown in Fig. 5(b). The wafer was then thinned to $\sim 100 \mu \mathrm{m}$ from the reverse side using a HF-nitric acid etching, as shown in Fig. 5(c). A photolithographic process was then used to pattern the backside of the wafer. A deep silicon etch from the backside completely removed the exposed silicon until the thermal oxide on the front side was exposed, as shown in Fig. 5(d). This etching process forms the flexible dielectric membrane connecting the imager plates. This etching process also separated each individual device from the substrate.

\section{Assembling the 3-D ARray}

After substrate release, the device was folded and assembled into a silicon prism under a magnifying glass. A manual assembly of the planar array into a hexagonal silicon prism took about $20 \mathrm{~min}$. It was found that the dielectric membrane can be bent more than $90^{\circ}$ without damaging the thin-film electrical interconnects sandwiched in the membrane. However, multiple back and forth bending larger than $90^{\circ}$ will break some of the electrical interconnects, and this results in an 
open circuit. After assembly, epoxy was used to fill the cavity inside the prism, such that the whole structure was glued in one piece. The filling materials are the $1: 1$ volumetric mixture of biphenyl epoxy resin (GC Electronics, Rockford, IL) and the hardener (polymercaptan). After the filling process, the 3-D imager prism was cured in an oven flushed with nitrogen gas for $30 \mathrm{~min}$ at $90{ }^{\circ} \mathrm{C}$. Fig. 6 is a photograph of a sevenimager prism after assembly. This device integrates one frontlooking annular imager and one six-side-looking planar imager array on a silicon prism for intravascular ultrasound imaging. The circular hole in the middle of the front-looking imager is for the guiding wire. The target operating frequencies of both front- and side-looking transducers on this device are $20 \mathrm{MHz}$. The 3-D imager prism was wire-bonded to a printed circuit board and is ready for testing. Hexagonal imager prisms with a width across the corner of hexagon, which is ranging from 1 to $4 \mathrm{~mm}$ in width and 2 to $4 \mathrm{~mm}$ in length, have been prototyped. The larger prisms were designed for operation at lower acoustic frequencies. In this design, the size of the imager array is approximately ten times the wavelength of the ultrasound transmitted from the transducer. Further miniaturization of the imager prism is possible with the transducers of higher frequencies.

\section{TRANSDUCER CHARACTERISTICS}

The transmitter characteristics of the transducers were tested using the setup shown in Fig. 7. The MEMS ultrasonic transducer array was soaked in a tank filled with either water or vegetable oil (Kroger vegetable oil, The Kroger Company, Cincinnati, OH). The MEMS ultrasonic transducers were driven by short electrical pulses generated by a pulse generator (Olympus 5077PR, Olympus NDT, Inc., Waltham, MA). The typical pulsewidth used in the transmission experiments ranged from 22 to $100 \mathrm{~ns}$, while the pulse amplitude ranged from 15 to $40 \mathrm{~V}$. A hydrophone (piezoceramic needle hydrophone TNU100A, NTR, Seattle, WA) was used in receiving the ultrasounds transmitted from the MEMS transducers. The hydrophone has a built-in 30-dB preamplifier. The output from the hydrophone was connected to a 40-dB amplifier (Olympus 5676, Olympus NDT, Inc., Waltham, MA) and, then, connected to an oscilloscope (HP 54624A, Palo Alto, CA) and a spectrum analyzer (Advantest R3261A, Advantest America, Inc., Santa Clara, CA).

Fig. 8 shows the ultrasound signals transmitted by a $46-\mu \mathrm{m}-$ diameter transducer upon excitation of an 80-ns-wide $35-\mathrm{V}$ electrical impulse without dc bias. Fig. 8(a) is the ultrasound signal and its spectrum with the transducer operating in water. The $-6-\mathrm{dB}$ fractional bandwidth is $13.3 \%$. Fig. 8 (b) is the ultrasound signal transmitted by the same transducer; however, the transducer was dipped in the vegetable oil before being placed in the water tank. In this case, the surface of the transducer is coated with a thin layer of vegetable oil. The purpose of the vegetable oil coating was to study the influence of an oil coating on acoustic performance of the transducer membrane. The $-6-\mathrm{dB}$ fractional bandwidth of the spectrum is $62 \%$. Fig. $8(\mathrm{c})$ is the ultrasound signal transmitted with the transducer operating in the vegetable oil. The $-6-\mathrm{dB}$ fractional bandwidth is $49.3 \%$. In

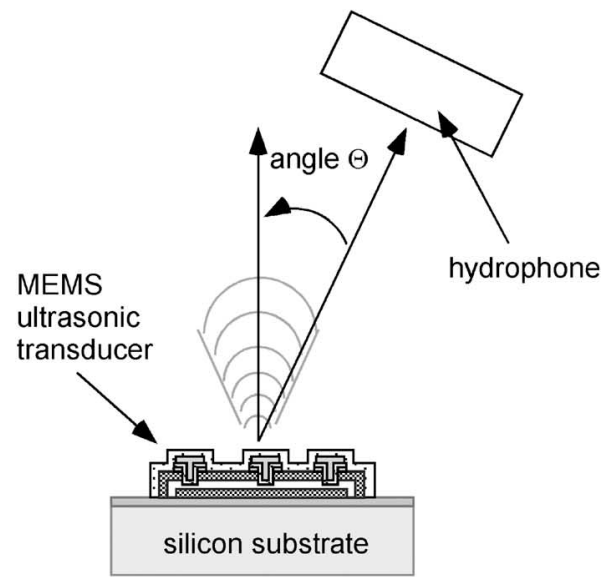

(a)

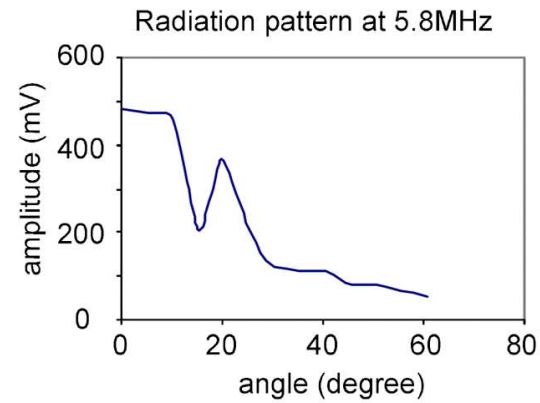

(b)

Fig. 9. (a) Experimental setup. (b) Radiation pattern of the 46- $\mu$ m-diameter transducer in water. The surface of the transducer is coated with a thin layer of vegetable oil.

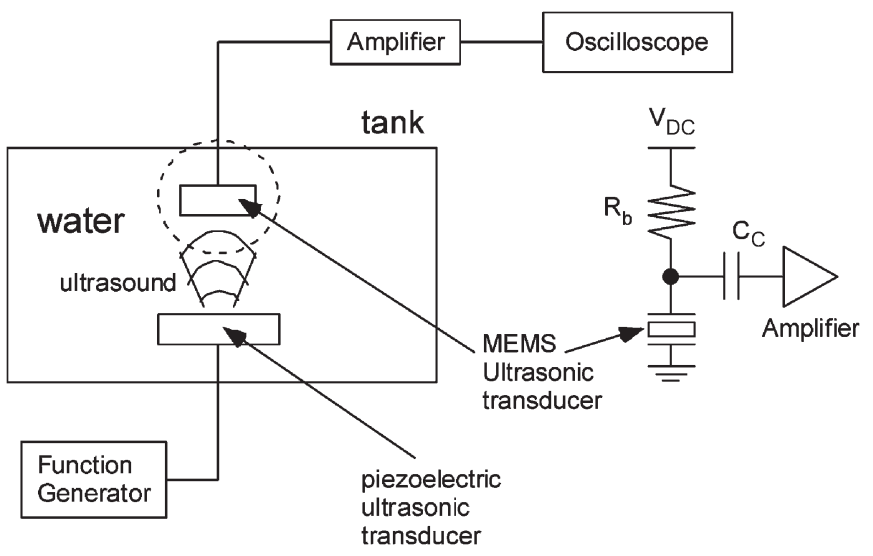

Fig. 10. Setup for receiver experiment.

water without vegetable oil coating on transducer, the central frequency was $5.3 \mathrm{MHz}$. In water with vegetable oil coating on transducer, the central frequency was $5.9 \mathrm{MHz}$. In vegetable oil, the central frequency shifted to $7.5 \mathrm{MHz}$. Fig. 9 shows the experimental setup and radiation pattern measurement result of a 46- $\mu$ m-diameter device in water. Similar to the experiment in Fig. 8(b), the transducer used in this radiation pattern measurement was coated with a thin layer of vegetable oil. The dip of amplitude near $15^{\circ}$ of the radiation pattern is due to Lamb wave crosstalk.

Fig. 10 shows the setup of the receiver characterization experiment. The experiments were conducted in a water tank, 


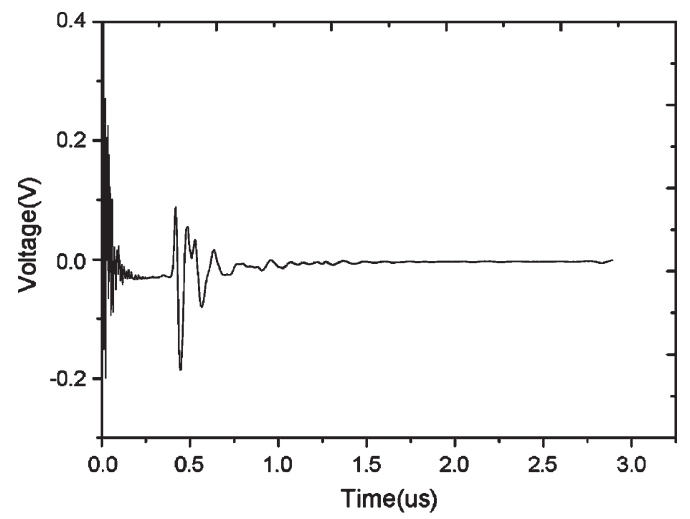

(a)

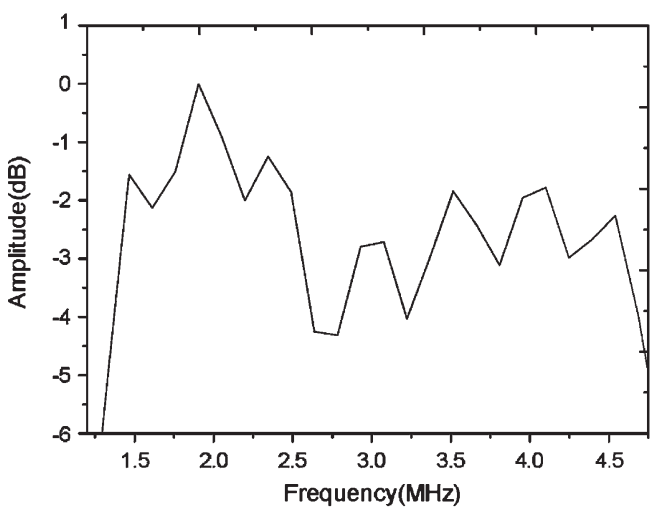

(b)

Fig. 11. (a) Ultrasound signal and (b) spectrum received by the $80-\mu \mathrm{m}$ diameter ultrasonic transducers. The experiment was conducted in water; however, the surface of the receiver MEMS ultrasonic transducer is coated with a thin layer of vegetable oil. The signal was after $80-\mathrm{dB}$ amplification.

with the MEMS ultrasonic transducer working as a receiver to receive ultrasounds that are transmitted from a commercial piezoelectric ultrasound transducer. The surface of the MEMS transducers was coated with a thin layer of vegetable oil by dipping the transducers into the vegetable oil before the experiment. A function generator (Agilent 33120A, Palo Alto, CA) was used in generating electrical pulses for triggering a piezoelectric ultrasonic transducer. The MEMS ultrasonic transducer was biased by a dc bias using an $R C$ circuit shown in Fig. 10. Fig. 11 is the ultrasound signal received by an $80-\mu \mathrm{m}-$ diameter MEMS transducer under $10-\mathrm{V}$ dc bias. The central frequency of the receiver spectrum was $2.0 \mathrm{MHz}$, and the $-6-\mathrm{dB}$ fractional bandwidth is $116 \%$.

\section{IMAGER CHARACTERISTICS}

Fig. 12(a) shows our experimental setup used in imaging with one of the CMUT arrays on the prism as the receiving transducer. A commercial pulser/receiver (Model 5073PR, Panametrics, Inc., Waltham, MA) was used in generating a negative pulse with a pulse energy of $104 \mu \mathrm{J}$. A copper wire phantom was placed in the focal zone of the transmitting transducer (V312, Panametrics, Inc., Waltham, MA), and the CMUT device was placed perpendicular to the transmitting transducer and facing the wire phantom. The transmitting transducer had

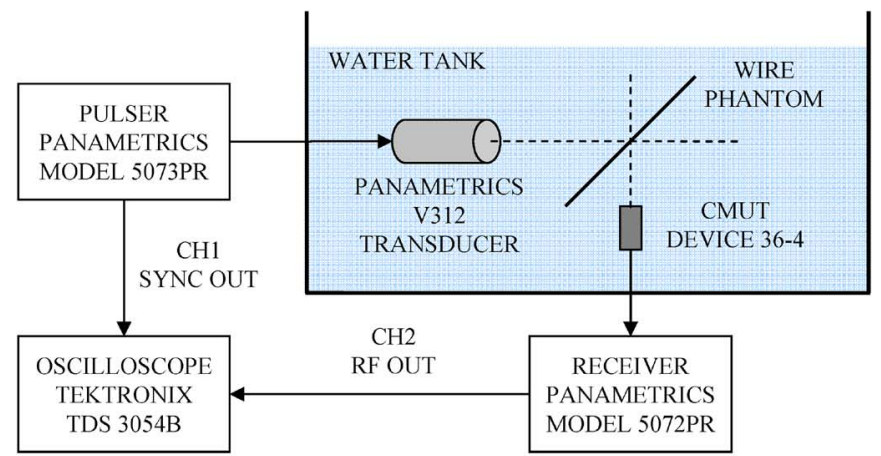

(a)

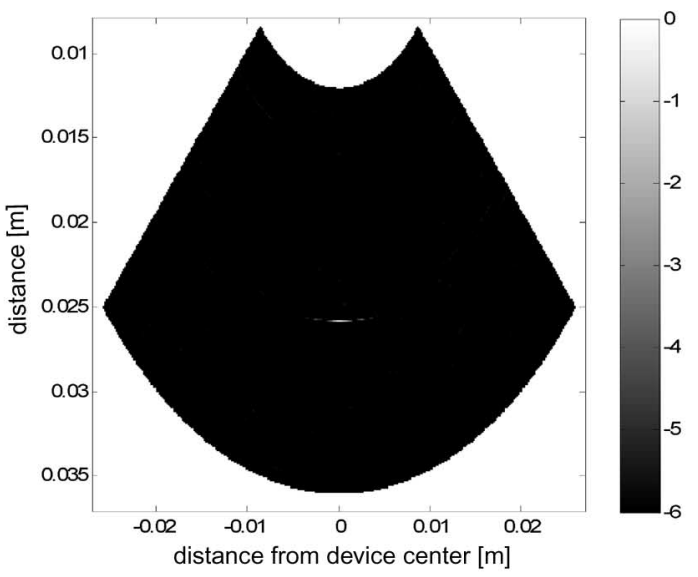

(b)

Fig. 12. (a) Experimental setup for image acquisition using the 3-D CMUT array. (b) Reconstructed B-mode image of a metal wire tip with a display dynamic range of $6 \mathrm{~dB}$.

a center frequency of $10 \mathrm{MHz}$. The signals were then high-pass filtered with a cutoff frequency of $1 \mathrm{MHz}$ and amplified with a 59-dB gain with another pulser/receiver (Model 5072PR, Panametrics, Inc., Waltham, MA). The RF signal was then digitized by an oscilloscope (TDS 3054B, Tektronix) and transferred to the computer. All postprocessing was done using MATLAB (The MathWorks Inc., Natick, MA).

A synthetic aperture focusing technique (SAFT) was employed for image reconstruction. That is, for every point in the image plane, geometric focusing delays (i.e., the time required for sound waves to propagate from the transmitting transducer to the image point and, then, to the receiving transducer) were applied. The delayed signals from all contributing array elements were then summed. A sector format with the viewing angle ranging from $-45^{\circ}$ to $45^{\circ}$ was adopted. The image is shown in Fig. 12(b). The measured $-3-\mathrm{dB}$ lateral beamwidth is $0.13 \mathrm{rad}$, and the measured $-3-\mathrm{dB}$ axial pulselength is $0.06 \mathrm{~mm}$. The CMUT device used for this imaging experiment was a 16-element linear array, which had an aperture size of $1.56 \mathrm{~mm}$ and an equal pitch of $97.5 \mu \mathrm{m}$. All 16 elements were used in SAFT. The signal-to-noise ratio (SNR) in the original experiments (CMUT was used as a receiver) was low ( $\sim 5 \mathrm{~dB})$; therefore, $-3-\mathrm{dB}$ beamwidth was used. However, if we switched to another setup (CMUT was used as a transmitter), the SNR can be improved to about $15 \mathrm{~dB}$. The measured 
-6-dB lateral beamwidth is $1.31 \mathrm{~mm}$, and the measured $-6-\mathrm{dB}$ axial pulselength is $1.3 \mathrm{~mm}$.

\section{CONCLUSION}

A miniature 3-D monolithic ultrasonic transducer array for internal imaging has been developed. Different from the traditional planar ultrasonic imagers that can view only in one direction, this 3-D array is able to view in multiple directions using seven planar imagers integrated on a hexagonal silicon prism. With this architecture, one flip-chip bonded or monolithically integrated front-end electronic circuit would be able to manage all the imagers on this 3-D array, and the number of bonding wires for connection to the external electronics can be reduced. Imager heads of diameter ranging from 1 to $4 \mathrm{~mm}$ and 2 to $4 \mathrm{~mm}$ in length have been prototyped to view in seven directions. Further miniaturization of the imager head is possible with higher frequency transducers. Preliminary testing shows that the imager transducers consistently behaved before and after the assembly process. This technology also enables a device with varying sizes of drum and allows the front- and side-looking elements to "see" at different wavelengths if desired. The advantages of the technology include size, monolithic, and multidirection. Applications of this 3-D imager array include capsule ultrasound endoscope, intravascular ultrasound, and other internal imaging needs.

\section{REFERENCES}

[1] P. Suetens, Fundamentals of Medical Imaging. Cambridge, U.K.: Cambridge Univ. Press, Mar. 2002.

[2] T. Szabo, Diagnostic Ultrasound Imaging: Inside Out. New York: Academic, 2004.

[3] R. A. Robb, Biomedical Imaging, Visualization, and Analysis. New York: Wiley-Liss, 1999.

[4] J. T. Bushberg, J. A. Seibert, E. M. Leidholdt, Jr., and J. M. Boone, The Essential Physics of Medical Imaging, 2nd ed. Philadelphia, PA: Lippincott Williams \& Wilkins, 2001.

[5] W. R. Hendee and E. R. Ritenour, Medical Imaging Physics. 4th ed., New York: Wiley-Liss, 2002.

[6] C. Frank, M. D. Dietrich, H. Allgayer, P. G. Archidiacono, H. D. Becker, and T. Beyer, Endoscopic Ultrasound: An Introductory Manual and Atlas. New York: Thieme Med., 2006.

[7] A. L. Baert and M. Oudkerk, Coronary Radiology (Medical Radiology/Diagnostic Imaging). New York: Springer-Verlag, 2004.

[8] C. L. de Korte, G. Pasterkamp, A. F. van der Steen, H. A. Woutman, and N. Bom, "Characterization of plaque components with intravascular ultrasound elastography in human femoral and coronary arteries in vitro," Circulation, vol. 102, no. 6, pp. 617-623, Aug. 2000.

[9] C. Z. Rosen, B. V. Hiremath, and R. E. Newnham, Piezoelectricity. New York: AIP Press, 1992.

[10] A. A. Vives, Piezoelectric Transducers and Applications. New York: Springer-Verlag, 2004.

[11] A. Nguyen-Dinh, L. Ratsimandresy, P. Mauchamp, R. Dufait, and M. Lethiecq, "High frequency piezo-composite transducer array designed for ultrasound scanning applications," in Proc. IEEE Ultrason. Symp., 1996, pp. 943-947.

[12] L. Ratsimandresy, P. Mauchamp, D. Dinet, N. Felix, and R. Dufait, "A $3 \mathrm{MHz}$ two dimensional array based on piezocomposite for medical imaging," in Proc. IEEE Ultrason. Symp., 2002, pp. 1265-1268.

[13] E. D. Light, S. F. Idriss, P. D. Wolf, and S. W. Smith, "Real time 3D intracardiac echocardiography," Ultrasound Med. Biol., vol. 27, no. 9, pp. 1177-1183, 2001.

[14] M. Greenstein, P. Lum, H. Yoshida, and M. S. Seyed-Bolorforosh, "A $2.5 \mathrm{MHz} 2 \mathrm{D}$ array with $Z$-axis electrically conductive backing," IEEE Trans. Ultrason., Ferroelectr., Freq. Control, vol. 44, no. 5, pp. 970-977, Sep. 1997.
[15] M. S. S. Bolorforosh, "Matching layer for front acoustic impedance matching of clinical ultrasonic transducers," U.S. Patent 5434827 , Jul. 18, 1995.

[16] Y. Huang, A. S. Ergun, M. H. Badi, and B. T. Khuri-Yakub, "Fabricating capacitive micromachined ultrasonic transducers with wafer-bonding technology," J. Microelectromech. Syst., vol. 12, no. 2, pp. 128-137, Apr. 2003.

[17] J. G. Knight and F. L. Degertekin, "Capacitive micromachined ultrasonic transducers for forward looking intravascular imaging arrays," in Proc. IEEE Ultrason. Symp., 2002, pp. 1079-1082.

[18] A. Bozkurt, I. Ladabaum, A. Atalar, and B. T. Khuri-Yakub, "Theory and analysis of electrode size optimization for capacitive microfabricated ultrasonic transducers," IEEE Trans. Ultrason., Ferroelectr., Freq. Control, vol. 46, no. 6, pp. 1364-1374, Nov. 1999.

[19] R. Carlton and A. M. Adler, "Fundamentals of medical imaging," in Principles of Radiographic Imaging: An Art and a Science, 3rd ed. Florence, KY: Thomson Delmar Learning, Sep. 2000.

[20] G. Maconi and G. B. Porro, Ultrasound of the Gastrointestinal Tract (Medical Radiology/Diagnostic Imaging). New York: Springer-Verlag, 2006.

[21] O. Oralkan, S. T. Hansen, B. Bayram, G. G. Yarahoglu, A. S. Ergun, and B. T. Khuri-Yakub, "CMUT ring arrays for forward-looking intravascular imaging," in Proc. IEEE Ultrason. Symp., 2004, pp. 403-406.

[22] Y. Wang, D. N. Stephens, and M. O'Donnell, "A forward-viewing ringannular array for intravascular imaging," in Proc. IEEE Ultrason. Symp., 2001, pp. 1573-1576.

[23] Y. Wang, D. N. Stephens, and M. O'Donnell, "Initial results from a forward-viewing ring-annular ultrasound array for intravascular imaging," in Proc. IEEE Ultrason. Symp., 2003, pp. 212-215.

[24] Y. Roh and B. T. Khuri-Yakub, "Finite element analysis of underwater capacitor micromachined ultrasonic transducers," IEEE Trans. Ultrason., Ferroelectr., Freq. Control, vol. 49, no. 3, pp. 293-298, Mar. 2002.

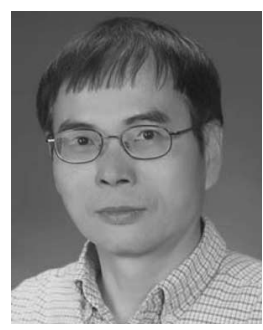

Jingkuang Chen received the B.S. and M.S. degrees in electrical engineering from the National Taiwan University, Taipei, Taiwan, R.O.C., and the Ph.D. degree in electrical engineering from the University of Michigan, Ann Arbor, in 1996. His Ph.D. dissertation focused on the development of microfluidic systems on silicon for selective drug delivery to the central nervous system and for inkjet printing.

From 1996 to 2004, he was a member of Research Staff with the Xerox Wilson Research Center, Webster, NY, working on the development of SOI MEMS optical systems and plastic microfluidic devices for printing applications. He joined the faculty of the University of New Mexico, Albuquerque, NM, in 2004, where he is currently an Associate Professor with the Department of Electrical and Computer Engineering. His research interests are invasive capacitive micromachined ultrasonic transducer arrays for diagnosis and treatment, porous silicon materials/devices as light source or biosensing element, and single-chip ultrasound-enhanced drug delivery system.

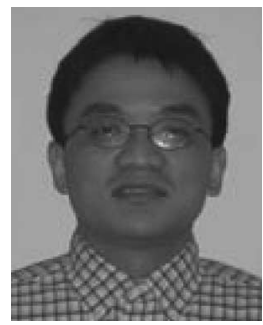

Xiaoyang Cheng received the B.S. degree in mechanical engineering from Huazhong University of Science and Technology, Wuhan, China, and the M.S. degree in electromechanical engineering from South China University of Technology, Guangzhou, China. He is currently working toward the Ph.D. degree in electrical engineering in the Department of Electrical and Computer Engineering, University of New Mexico, Albuquerque, NM.

His research interests include the design, fabrication, and characterization of invasive MEMS capacitive micromachined ultrasonic transducers for biomedical applications. 


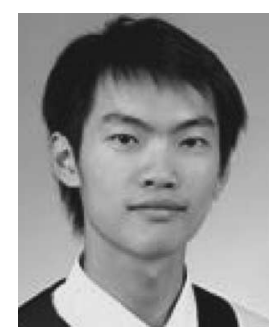

I-Ming Shen was born in Taichung, Taiwan, R.O.C, in 1983. He received the B.S. degree in electrical engineering from the National Taiwan University, Taipei, Taiwan, R.O.C., in 2005. He is currently working toward the M.S. degree in electrical engineering in the Department of Electrical Engineering, National Taiwan University.

His current research interests include biomedical ultrasound imaging, especially for beam forming and signal processing.

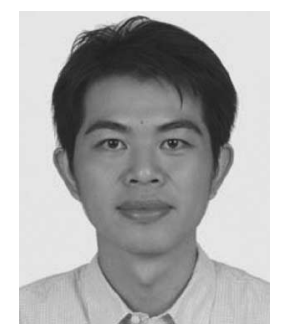

Jian-Hung Liu received the B.S. degree in electrical engineering from Southern Taiwan University of Technology, Tainan, Taiwan, R.O.C., in 2002 and the M.S. degree in bioengineering from the National Yang-Ming University, Taipei, Taiwan, R.O.C., in 2004. He is currently working toward the Ph.D. degree in electrical engineering in the Department of Electrical Engineering, National Taiwan University, Taipei.

His current research interests include highfrequency small-animal imaging and high-frequency array transducers.

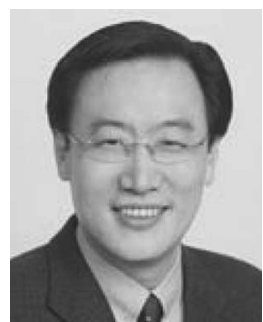

Pai-Chi Li (S'93-M'95-SM'01) received the B.S. degree in electrical engineering from the National Taiwan University, Taipei, Taiwan, R.O.C., in 1987 and the M.S. and Ph.D. degrees in electrical engineering: systems from the University of Michigan, Ann Arbor, in 1990 and 1994, respectively.

From 1990 to 1994, he was a Research Assistant with the Department of Electrical Engineering and Computer Science, University of Michigan. He joined Acuson Corporation, Mountain View, CA, as a member of Technical Staff in June 1994, working primarily on medical ultrasonic imaging system designs for both cardiology and general imaging applications. He joined the National Taiwan University in August 1997, where he is currently a Professor with the Department of Electrical Engineering and the Director of the Graduate Institute of Biomedical Electronics and Bioinformatics. He is also with the editorial board of Ultrasonic Imaging. His current research interests include biomedical ultrasonic imaging and signal processing.

Dr. Li received the Distinguished Achievement Award from the University of Michigan in 1994 for his outstanding academic achievement in electrical engineering: systems. He also received the Dr. Wu Dayou Research Award from the National Science Council, the Outstanding Young Electrical Engineer Award from the Chinese Institute of Electrical Engineering, and the Distinguished Industrial Collaboration Award from the Ministry of Education in 2002, the Outstanding Researcher Award from the National Taiwan University in 2003, the Distinguished Research Award from the National Science Council in 2004, the Distinguished Research Achievement Award from the National Taiwan University in 2004, and the Outstanding Electrical Engineering Professor Award from the Chinese Institute of Electrical Engineering in 2005. He is an Associate Editor for the IEEE TRANSACTIONS ON UlTRASONICS, FERROELECTRICS, AND FREQUENCY CONTROL.

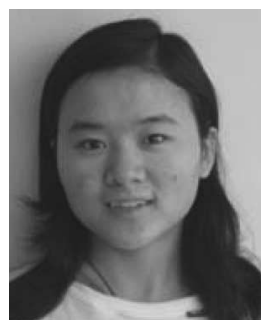

Mengli Wang received the B.S. degree in electrical engineering from Anhui University of Technology, Anhui, China, in 2002 and the M.S. degree in electrical engineering from Tianjin University, Tianjin, China, in 2005. She is currently working toward the Ph.D. degree in electrical engineering in the Department of Electrical and Computer Engineering, University of New Mexico, Albuquerque, NM.

Her research interests are the testing and application of capacitive micromachined ultrasonic transducers. 